PAPERS

\title{
Meyer-Neldel rule in the conductivity of phase separated manganites
}

\author{
Jacek Przybytek $^{* * *}$, Vladimir Markovich ${ }^{* * *}$, Grzegorz Jung $^{* * *, * * * *}$
}

\begin{abstract}
Meyer-Neldel behaviour of the conductivity of phase separated $\mathrm{La}_{1-x} \mathrm{Ca}_{x} \mathrm{MnO}_{3}$ manganite system in the low Ca-doping range has been investigated. Evolution of the isokinetic temperature of the conductivity, modified by Ca-doping, hydrostatic pressure and current bias has been determined. In addition, the evolution of the isokinetic temperature with ageing has also been studied. It is found that the Meyer-Neldel behaviour of the manganite system stems from multi-excitation entropy mechanism. The isokinetic temperatures estimated from pressure and doping effects coincide but differ from those determined using current and ageing controlled conductivity changes. It is concluded that in the presence of a detailed theoretical model of the excitations coupling in manganites, the investigations of the Meyer-Neldel effect may became a powerful tool for characterization and investigation of transport mechanisms in phase separated manganites.
\end{abstract}

K e y w ord s: manganites, Meyer-Neldel rule, activation energy, metastable resistivity

\section{Thermally activated conductivity and Meyer-Neldel rule}

Electrical conductivity of phase separated manganites is governed by thermal activation in several relatively wide temperature ranges. In this type of processes, the system must overcome an energy barrier in order for the process to go forward. The rate of thermally activated processes increases exponentially with temperature $T$, as described by the well-known empirical Arrhenius relationship (1):

$$
k=A \exp \left(-E_{\mathrm{a}} / k_{B} T\right),
$$

where $E_{\mathrm{a}}$ is the activation energy, $k_{\mathrm{B}}$ is Boltzmann's constant, and $A$ the pre-exponential factor. The theoretical expression for the rate constant, known as the Eyring equation, results from the transition state theory [1] and reads:

$$
k=\frac{\kappa k_{B} T}{h} \exp \left(-\frac{d G^{* *}}{k_{B} T}\right)
$$

Here, $h$ is Planck's constant, $\kappa$ is the transmission coefficient, often assumed to be equal to one, $d G^{* *}=$ $d H^{* *}-T d S^{* *}$ is the Gibbs free energy of activation, related to the activation enthalpy $d H^{* *}$ and activation entropy $d S^{* *}$. The activation parameters of the Arrhenius and Eyring equations are related as $E_{\mathrm{a}}=d H^{* *}+k_{B} T$, and $A=\left(\kappa k_{B} T / h\right) \exp \left(d S^{* *} / k_{B}\right)$. In practice, the differences between Arrhenius activation energy $E_{\mathrm{a}}$ and the activation enthalpy $d H^{* *}$ are quite small and these two energies are frequently used interchangeably.

When the rate constant $k$ obeys the Arrhenius' equation, a plot of the logarithm of the rate $\ln (k)$ against the inverse temperature $(1 / T)$ produces a straight line, referred to as the Arrhenius plot, see Fig. 1. The slope of the Arrhenius plot can be used to find the activation energy, while the $y$-intercept of the extrapolated line gives the pre-exponential factor $A$.

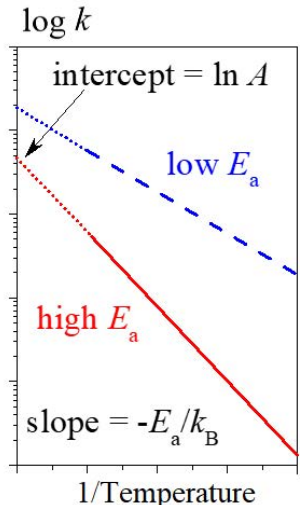

$1 /$ Temperature

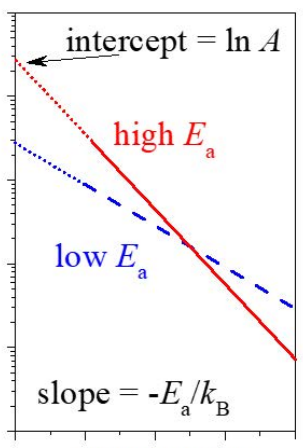

$1 /$ Temperature
Fig. 1. Arrhenius plot for two different activation energies with preexponencial factor: left - decreasing, and right - increasing, with increasing activation energy

The role of the pre-factor $A$ in a thermally activated process is frequently underestimated. It is often assumed that increase of the activation energy $E_{\text {a }}$ always decreases the rate of the thermally activated process. This however is true, as illustrated in Fig. 1, only when increase of the activation energy does not influence, or is accompanied by a decrease of the associated exponential pre-factor $A$. In the opposite case, when increase of the activation energy

*Institute of High Pressure Physics PAS, 01-142 Warsaw, Poland, jacek.przybytek@fuw.edu.pl, **Institute of Experimental Physics, Faculty of Physics, University of Warsaw, 02-093 Warsaw, Poland, ***Department of Physics, Ben Gurion University of the Negev, 84105 Beer Sheva, Israel, ****Institute of Physics PAS, 02-668 Warsaw, Poland 


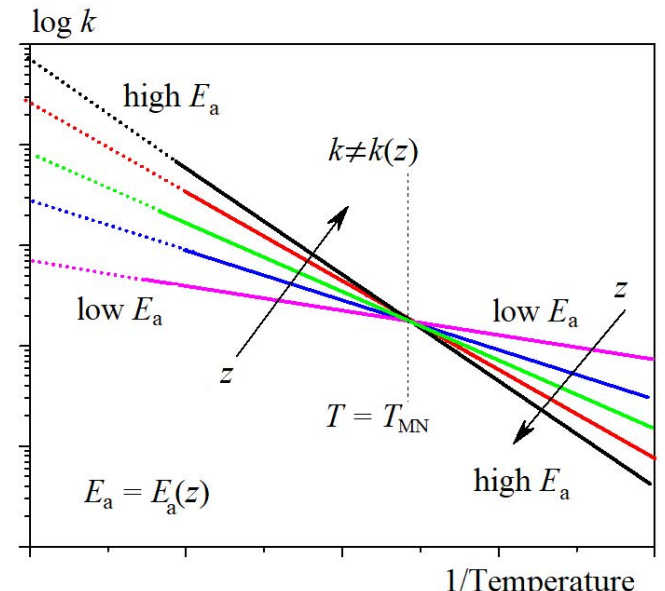

Fig. 2. Arrhenius plot for a process obeying NMR and activation energy depending on an experimental parameter $z$

is accompanied by an increase of the exponential prefactor $A$, the rate of the process decreases with increasing $E_{\text {a }}$, only at low temperatures, while at high temperatures an increase of the rate will be observed, see Fig. 1.

Meyer and Neldel determined empirically that in the thermally activated electrical conductivity of some oxide semiconductors the pre-exponential factor increases exponentially in proportion to the thermal activation energy [2]. As a consequence, a decrease of the rate, due to an increase in the activation energy, can be partially compensated by an opposite change of the pre-exponential factor. Such compensation effect was in fact originally derived in chemical kinetics and latter observed in many processes in chemistry, condensed matter physics, biology, and geology [3]. The expression for the thermally activated rate accounting for this phenomenon, known as the Compensation Law, the Isokinetic Rule, or the Meyer-Neldel Rule (MNR), reads

$$
k=A_{00} \exp \left(E_{\mathrm{a}} / k_{B} T_{M N}\right) \exp \left(-E_{\mathrm{a}} / k_{B} T\right)
$$

where $A_{00}$ and $T_{\mathrm{MN}}$ are constants for a given process. It follows, that in a system obeying the Meyer-Neldel rule, an experimental parameter $z$ capable of changing the activation energy, does not influence the rate of a thermally activated process at the temperature $T=T_{\mathrm{MN}}$, see Fig. 2 .

A consistent theoretical explanation of the compensation effect in rich variety of physical systems, including also the non-solid state systems, is provided by a general multi-excitation entropy (MEE) thermodynamic model [3]. Observe that MNR rate (3) has in fact a form of the Eyring equation. Since the kinetic behaviour of a system depends upon the free energy rather than on the energy or the enthalpy alone, the MNR behaviour will appear whenever the activation entropy becomes proportional to the activation enthalpy. This happens when an activation energy is large and the thermally activated process needs to acquire big number of individual excitations to overcome a barrier. Large number of activations can be assembled out of the total number of available excitations in many ways, what increases the activation entropy $S^{* *}$. The number of excitations needed to overcame the barrier equals to the activation enthalpy $H^{* *}$ divided by the energy of an individual excitation. In the limit of large number of excitations, the activation entropy becomes proportional to the enthalpy and leads to MNR behaviour. In the framework of the MEE model the characteristic Meyer-Neldel temperature is determined by the energy of an individual excitation divided by the factor $k_{\mathrm{B}} \ln (N)$, where $N$ is a coupling constant measuring the number of available excitations within an interaction volume. Thus, whenever the activation energy is large with respect to thermal excitations, the MNR behaviour should appear [3].

\section{Electrical conductivity of manganite's single crystals}

Mixed valence manganese oxides $\mathrm{R}_{1-x} \mathrm{~A}_{x} \mathrm{MnO}_{3}$ ( $\mathrm{R}$ is rare-earth cation, $\mathrm{A}$ is alkali or alkaline earth cation) show a variety of interesting crystallographic, electronic and magnetic properties due to a pronounced phase separation (PS), consisting in dynamic coexistence of phases with different orbital, magnetic, and electronic properties [4]. The unusual consequences of phase separation in manganites include the colossal magnetoresistive effect (CMR) and appearance of peculiar long and short life-time metastable states with different resistivity. The metastable states appear either spontaneously, or became induced by changing external parameters such as temperature, magnetic field or electrical bias [5-9]. The nature of physical mechanisms responsible for this metastability are still not fully understood.

The primary factor influencing properties of doped lanthanum manganites is the doping level $x[4,10]$. In the parent compound, $\mathrm{LaMnO}_{3}, \mathrm{Mn}$ is in a $3+$ valence state. Upon doping by a divalent element $\left(\mathrm{Ca}^{2+}, \mathrm{Sr}^{2+}, \mathrm{Ba}^{2+}\right.$, $\mathrm{Pb}^{2+}$ ) the $\mathrm{Mn}$ is driven into a mixed valence state $\mathrm{Mn}^{3+} / \mathrm{Mn}^{4+}$. For $\mathrm{La}_{1-x} \mathrm{Ca}_{x} \mathrm{MnO}_{3}$ (LCMO) manganite, the critical doping level $x_{\mathrm{c}}=0.225$ separates the ferromagnetic and metallic (FMM) ground state, seen above $x_{\mathrm{c}}$, from the orbital ordered ferromagnetic and insulating (FMI) ground state below $x_{\mathrm{c}}$. When the hole-doping level decreases below $x=0.125$, the LCMO ground state becomes insulating and antiferromagnetic. In the low doping range $0.125<x<0.225$, an additional insulating ferromagnetic phase, incompatible with the double exchange mechanism, coexists with the ferromagnetic metallic phase and strongly influences transport properties of LCMO [11]. With increasing temperature, the FMM phase undergoes a concomitant metal-to-insulator (M-I) and FM (ferromagnetic)-to-PM (paramagnetic) transitions. 


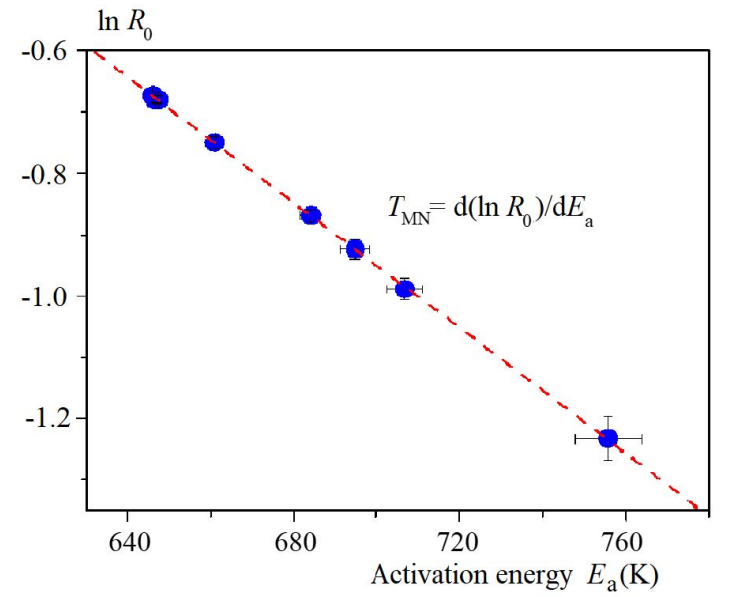

Fig. 3. An example of resistance of the manganite crystal following the Meyer-Neldel rule

Meyer-Neldel behaviour in electrical conductivity was reported for several strongly disordered systems. However, to our best knowledge, the MNR behaviour of colossal magnetoresistive materials has been reported only once, in thin films of $\operatorname{Pr}_{0.68} \mathrm{Ca}_{0.32} \mathrm{MnO}_{3}$ with different degrees of octahedral disorder [12]. Here we present evidence for MNR behaviour of the electrical conductivity of LCMO manganite bulk single crystals.

\section{Samples and measuring procedures}

In the experiments we have employed LCMO single crystals grown by means of the floating zone technique in an optical furnace, from sintered ceramic rods of high purity precursors. The chemical composition of the crystals was determined by field emission scanning electron microscopy and cross-checked by scanning electron microscopy and EDX analysis. Phase analysis of the crystals was performed at room temperature by X-ray powder diffraction. The details of the fabrication procedures as well as the results of the structural, chemical, resonance, and magnetic characterization of the employed crystals were published elsewhere [5-8].

Samples for the transport measurements in the form of $1 \mathrm{~mm}$ thick and $3 \mathrm{~mm}$ wide bars directed along the [100] crystalline direction, were cut off from the blocks of the well-defined orientation in the as-grown crystals. Transport characteristics were measured in a standard four-point contact arrangement, with $0.33 \mathrm{~mm}$ distance between the voltage contacts. Current and voltage leads were indium soldered to the vacuum evaporated gold contacts. Voltages developing across current biased sample were amplified by very low noise room temperature preamplifier located on the top of the cryostat and processed by a computer. The resistivity characteristics were measured using alternatively, ac lock-in or dc current techniques. Measurements of the resistance under hydrostatic pressure were carried out in a $\mathrm{CuBe}$ pressure cell.

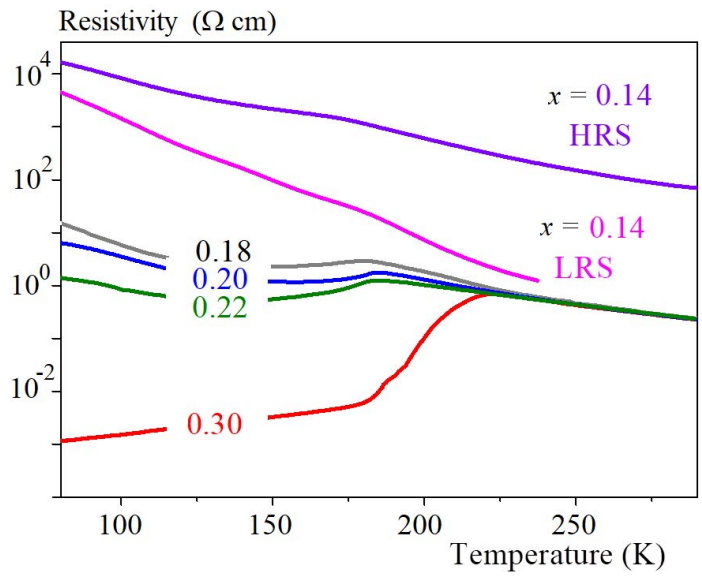

Fig. 4. Temperature dependence of resistivity of $\mathrm{La}_{1-x} \mathrm{Ca}_{x} \mathrm{MnO}_{3}$ single crystals at different level of Ca-doping $x$

The temperature was measured by copper-constantan thermocouple attached to the cell, while the pressure was monitored by a manganin gauge.

\section{Meyer-Neldel rule in conductivity of manganite single crystals: Experimental results}

We have investigated temperature dependence of the resistance $R(T)$ of the mixed valance $\mathrm{La}_{1-x} \mathrm{Ca}_{x} \mathrm{MnO}_{3}$ manganite with different Ca-doping levels $x$. In addition, for two fixed doping levels $(x=0.18$ and $x=0.14)$ we have also investigated the evolution of the $R(T)$ characteristics with changing bias current, hydrostatic pressure, and ageing.

A set of $R(T)$ characteristics recorded for differently doped crystals is shown in Fig. 4. Labels HRS and LRS in the $x=0.14$ recordings refer to the high and low metastable resistivity states. As fabricated $\mathrm{La}_{0.86} \mathrm{Ca}_{0.14} \mathrm{MnO}_{3}$ crystals was found to be in the high resistivity state. However, already during the very first thermal cycles between liquid He and room temperatures, accompanied by bias current sweeps, the pristine HRS spontaneously evolved into the low resistivity metastable state, as discussed in details elsewhere $[9,13]$. The LRS was characterized by a long lifetime and persisted for more than three months of continuous experimentation. However, after two months break in the experiments, the crystal spontaneously returned to the HRS state, which then evolved further towards even higher resistivity during long term ageing.

Similar spontaneous and current induced metastable resistivity states were also observed in LCMO crystals and thin films with other doping levels from $0.125<x<$ 0.225 range [5-8]. However, since $x=0.14$ LCMO crystals exhibits most pronounced differences between HRS and LRS states [13], we have focused our investigations of the MNR effect in metastable states and ageing on $x=0.14$ doping level only. 
Table 1. Summarize of results

\begin{tabular}{lcc} 
& $\begin{array}{c}T_{\mathrm{MN}} \text { from } R(T), \\
\text { data below } T_{\mathrm{C}}\end{array}$ & $\begin{array}{c}T_{\mathrm{MN}} \text { from } R(T), \\
\text { data above } T_{\mathrm{C}}\end{array}$ \\
\hline Doping controlled conductivity & $228.3 \pm 5.1 \mathrm{~K}$ & $228.3 \pm 5.1 \mathrm{~K}$ \\
Pressure controlled conductivity for $x=0.18$ & $228.0 \pm 5.0 \mathrm{~K}$ & $228.0 \pm 5.0 \mathrm{~K}$ \\
Bias controlled conductivity for $x=0.18$ & $116.3 \pm 1.7 \mathrm{~K}$ & $205.8 \pm 2.0 \mathrm{~K}$ \\
Bias controlled conductivity for $x=0.14$, HRS state & $178.2 \pm 0.7 \mathrm{~K}$ & $178.2 \pm 0.7 \mathrm{~K}$ \\
Bias controlled conductivity for $x=0.14$, aged HRS state & $182.5 \pm 2.4 \mathrm{~K}$ & $238.7 \pm 2.9 \mathrm{~K}$ \\
\hline
\end{tabular}

For all temperature ranges where the resistivity is thermally activated, ie increases with decreasing temperature in an exponential way, we have locally fitted the resistance version of the MNR equation (3),

$$
\begin{array}{r}
R(T)=R_{0} \exp \left(-E_{\mathrm{a}} / k_{B} T\right)= \\
R_{00} \exp \left(E_{\mathrm{a}} / k_{B} T_{M N}\right) \exp \left(-E_{\mathrm{a}} / k_{B} T\right)
\end{array}
$$

to the experimental $R(T)$ characteristics and from the fit determined the local activation energy

$$
E_{\mathrm{a}}(T)=\frac{d[\ln R(T)]}{d\left(1 / k_{B} T\right)}
$$

and the associated exponential pre-factor $R_{0}$. We have then plotted the logarithm of the pre-exponential factor $R_{0}$ as the function of the activation energy $E_{\mathrm{a}}$. This is illustrated in an example shown in Fig. 3.

According to the equations (3) and (4), the hallmark of the MNR behaviour is the linear dependence of the logarithm of the pre-exponential factor on the activation energy. The values of the isokinetic temperature $T_{\mathrm{MN}}$ can be determined from the slope of the linear plots. Thus determined $T_{\mathrm{MN}}$ should be obviously consistent with the temperature at which the process becomes independent of the experimental parameter that changes the resistivity.

Our experiments showed that the resistivity of lowdoped LCMO manganites influenced by doping, applied pressure, bias current, and ageing time, follows the Meyer-Neldel compensation rule in almost all studied cases. The only deviations from MNR were observed in the temperature ranges at which the local activation energy becomes comparable or smaller than the thermal activation energy $k_{\mathrm{B}} T$, what clearly violates the prerequisites of the multiple excitation entropy model [3]. The results of our investigations are summarized in Tab. 1.

\section{Discussion}

The fact that the MNR behaviour breaks down whenever the local activation energy becomes smaller that the thermal excitation $k_{\mathrm{B}} T$, indicates that the MeyerNeldel rule in LCMO single crystals stems from the multi-excitation entropy mechanism. Multiple excitation entropy model describes the Meyer-Neldel temperature
$T_{\mathrm{MN}}$ only as a function of a generic coupling constant that measures the number of available excitations within the interaction volume [3]. In-depth analysis of our experimental results would require a comprehensive theoretical model relating the coupling constant to all possible transport mechanisms in the investigated manganite system. Regrettably, such model is not available at present. However, even in the absence of the model, some valuable conclusions can be drawn from the experimental results.

The Meyer-Neldel temperature revealed from the measurements of differently doped LCMO crystals coincides exactly with $T_{\mathrm{MN}}$ obtained from pressure dependent resistivity of LCMO crystal doped at $x=0.18$. This is consistent with the fact that both doping and pressure influence the resistivity of mixed valance manganites through a similar mechanism of enhancing concentration of itinerant electrons and the hopping rate, which favors charge delocalization and increases ferromagnetic interactions [14]. On the other hand, $T_{\mathrm{MN}}$ determined from bias dependent resistivity differs from that obtained in pressure and doping experiments, what suggests that bias current influences conductivity of the studied manganite through a markedly different mechanism. This is confirmed by differences between temperature dependencies of activation energies $E_{\mathrm{a}}(T)$, derived using pressure and current bias influenced resistance of $x=0.18 \mathrm{LCMO}$, as shown in Fig. 5 and Fig. 6.

At a first glance the overall shape of both dependencies is similar. At high temperatures, the activation energy is almost temperature independent, with exception of the small local maximum associated with Jahn-Teller transition. With decreasing temperature, there is a sharp drop in the activation energy at $T=T_{\mathrm{C}}$, associated with strong increase of ferromagnetic correlations at the PMto-FM phase transition [4].

Negative values of the activation energy at temperatures close to $T_{\mathrm{C}}$ are only related to the procedure of determining $E_{\mathrm{a}}$ and have no physical meaning. Nevertheless, there are marked differences between the dependencies. First of all, dc current changes the activation energy in a much weaker way than the applied pressure. Moreover, current bias does not change significantly any of the characteristic temperatures, which, on the other hand, are quite sensitive to the applied pressure. With increasing pressure, the Jahn-Teller maximum decreases and moves towards lower temperatures, consistently with 


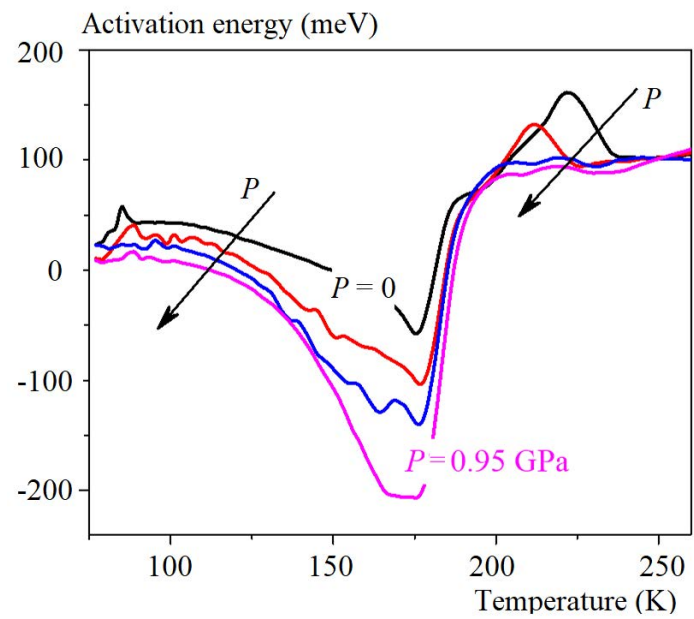

Fig. 5. Temperature dependence of the local activation energy of $\mathrm{La}_{0.82} \mathrm{Ca}_{0.18} \mathrm{MnO}_{3}$ single crystal at increasing applied hydrostatic pressure

Activation energy (meV)

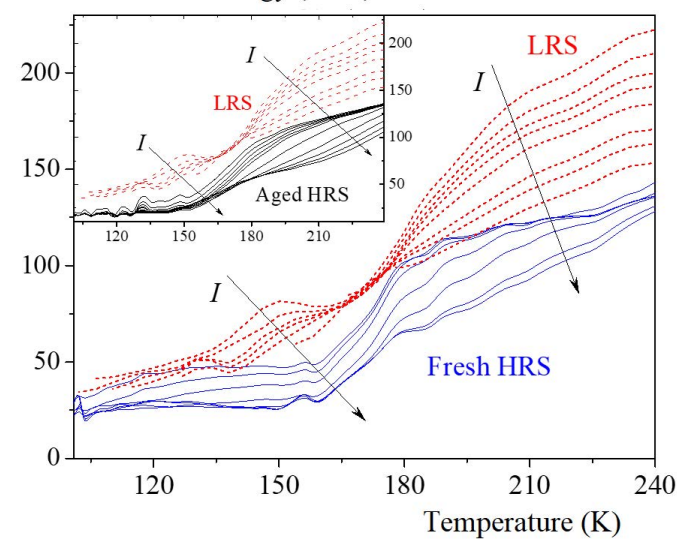

Fig. 7. Temperature dependence of the local activation energy of $\mathrm{La}_{0.82} \mathrm{Ca}_{0.18} \mathrm{MnO}_{3}$ single crystal in various resistivity states at different bias currents

the known sharp decrease of the temperature of JahnTeller transition with increasing doping [10]. Increasing pressure also increases the PM-FM transition temperature, similarly to $T_{C}$ increase with increasing doping.

It can be therefore concluded that current enforced changes of the resistivity are indeed due to a different mechanism than pressure induced changes. Applied pressure influences the resistivity by increasing the number of itinerant electrons, what causes an increase of ferromagnetic correlations. Increasing volume of the FMM phase results in a reduction of the energy gap, decrease of the Jahn-Teller transition temperature, shifting of the resistivity upturn point in the $R(T)$ characteristics to lower temperatures, and increase of the Curie temperature. Nonetheless, there is no consensus in the existing literature on the physics behind the bias dependent resistivity of mixed valence manganites. One of the plausible scenario may consist in melting of the current driven high density polarons, accumulating at phase boundaries between metallic and insulating domains, into low density
Activation energy (meV)

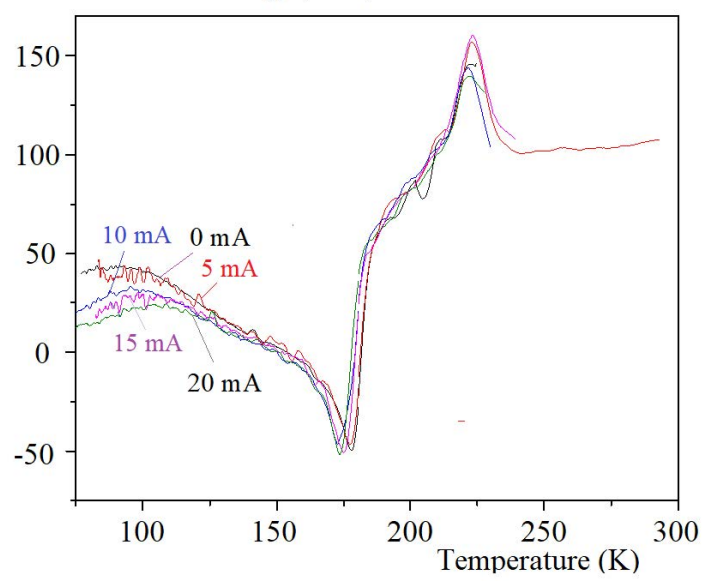

Fig. 6. Temperature dependence of the local activation energy of $\mathrm{La}_{0.82} \mathrm{Ca}_{0.18} \mathrm{MnO}_{3}$ single crystal at increasing bias current

and high mobility free electrons [15]. Additional features, such as differences in Meyer-Neldel temperatures below and above $T_{\mathrm{C}}$ may be attributed to different conduction mechanism at low (ferromagnetic) and high (paramagnetic) temperature ranges.

Physical mechanism behind metastable resistivity states in mixed valance manganites remains to be obscure. Therefore, any additional insight into the physics of metastable states cannot be underestimated. Our experiments reveal that a transition of the $\mathrm{La}_{0.86} \mathrm{Ca}_{0.14} \mathrm{MnO}_{3}$ manganite from the low resistivity metastable state to the high resistivity one is accompanied by an increase of the Meyer-Neldel temperature. The ageing of the metastable state causes further $T_{\mathrm{MN}}$ increase. The discussion of this behaviour in terms of increasing coupling constant is not be productive, in the light of missing proper microscopic model describing transport governed by multi-excitation entropy in mixed valence manganite systems. However, we can draw a straightforward phenomenological conclusion from the analysis of $T_{\mathrm{MN}}$ data and from ageing of the bias current dependence of the local activation energy shown in Fig. 7. In the pristine high resistivity state HRS, low resistivity metastable state LRS, and in the aged HRS state at different bias currents. One notes that bias current dependence of the activation energy in the crystal Ca-doped at $x=0.14$ is much stronger than in the $x=0.18$ crystal and that there is a systematic decrease of the activation energy with increasing age of the sample.

It follows from the comparison of the Eyring equation (2) with the MNR equation (3) that $T_{\mathrm{MN}}$ is simply a proportionality constant between the activation enthalpy $H^{* *}$ and activation entropy $S^{* *}$. We have pointed out earlier that differences between the activation enthalpy and activation energy $E_{\mathrm{a}}$ are practically negligible. Therefore, one can write that $E_{\mathrm{a}}=T_{\mathrm{MN}} S^{* *}$. Since the activation energy decreases with ageing, the increase of Meyer-Neldel temperature with ageing time indicates 
that the entropy of the system has to decrease with ageing of the sample. In other words, the higher resistance metastable states are more ordered. Obviously, this conclusion does not violate the II thermodynamics law because the investigated system is not an insulated one. On the other hand, the conclusion of the progressive ordering of the metastable state concurs with results of our independent noise investigations of the metastable resistivity states $[9]$.

\section{Conclusions}

In summary, we have shown that conductivity of mixed valance Ca-doped manganite single crystals obeys the Meyer-Neldel rule in a wide range of temperatures and under changing electric bias, hydrostatic pressure, doping, and ageing. The behaviour of characteristic MeyerNeldel temperature $T_{\mathrm{MN}}$ revealed significant differences between mechanisms of resistivity changes enforced by doping or pressure and current bias or ageing. Conductivity changes with changing doping and applied pressure are caused by the same mechanism of enhancing $e_{\mathrm{g}}$ conduction electrons. Bias influences the conductivity through a different mechanism, likely consisting in melting of small polarons into free carriers at phase-separated interfaces.

Meyer-Neldel temperature $T_{\mathrm{MN}}$ of the high resistivity metastable state is higher than in the low resistivity one. Systematic increase of the $T_{\mathrm{MN}}$ with ageing brought us to the conclusion that resistivity increase in aged metastable states consists in ordering of the electronic state of the manganite system.

It can be concluded that Meyer-Neldel temperature can constitute an excellent parameter enabling one to monitor and characterize changes in the conductivity associated with metastable resistivity states, provided a microscopic model relating electrical transport in mixed valence manganite systems with multi excitation entropy conditions will be further developed in the future [16].

\section{Acknowledgements}

This research was supported by the Polish NCN grant 2012/05/B/ST3/03157. The crystals used in the experiments were fabricated by J. Fink-Finowicki in the Institute of Physics in Warsaw and by Ya. Mukovskii in the Moscow State Steel and Alloys Institute.

\section{REFERENCES}

[1] M. G. Evans and M. Polanyi, "Some applications of the transition state method to the calculation of reaction velocities, especially in solution", Trans. Faraday Soc., vol. 31, pp. 875-894, 1935.
[2] W. Meyer and H. Neldel, "Concerning the relationship between the energy constant epsilon and the quantum constant alpha in the conduction-temperature formula in oxydising semi-conductors", Physikalische Zeitschrift, vol. 38, pp. 1014-1019, 1937..

[3] A. Yelon, B. Movaghar, and R. S. Crandall, "Multi-excitation entropy: its role in thermodynamics and kinetics", Reports on Progress in Physics, vol. 69, pp. 1145-1194, 2004.

[4] E. Dagotto, "Nanoscale Phase Separation and Colossal Magnetoresistance", Springer series in Solid State Physics, Springer -Verlag, Berlin, 2003.

[5] Y. Yuzhelevski, V. Markovich, V. Dikovsky, G. Gorodetsky, G. Jung, D. A. Shulyatev, and Ya. M. Mukovskii, "Current-Induced Metastable Resistive States and Memory Effects in Low-Doped Manganites", Phys. Rev. B, vol. 64, pp. 224428, 2001.

[6] V. Markovich, G. Jung, Y. Yuzhelevski, G. Gorodetsky, A. Szewczyk, M. Gutowska, D. A. Shulyatev, and Ya. M. Mukovskii, "Electric Field/Current Induced Metastability and Resistivity Relaxation in $\mathrm{La}_{0.8} \mathrm{Ca}_{0.2} \mathrm{MnO}_{3}$ at Low Temperatures", Phys. Rev. B, vol. 70, pp. 064414, 2004.

[7] V. Markovich, G. Jung, Y. Yuzhelevskii, G. Gorodetsky, F. X. $\mathrm{Hu}$, and J. Gao, "Resistivity of $\mathrm{La}_{0.8} \mathrm{Ca}_{0.2} \mathrm{MnO}_{3}$ Manganite Thin Films", Phys. Rev. B, vol. 75, pp. 104419, 2007.

[8] E. Bose, S. Karmakar, B. K. Chaudhuri, S. Pal, "Importance of double-exchange interaction in low-doped ferromagnetic insulator", Sol. State Commun., vol. 145, pp. 149-153, 2008.

[9] J. Przybytek, J. Fink Finowicki, R. Puzniak, V. Markovich, and G. Jung, "Noise signatures of metastable resistivity states in ferromagnetic insulating manganite", J. Appl. Phys., vol. 118, pp. 043903, 2015.

[10] J. B. Goodenough, "Handbook on the Physics and Chemistry of Rare Earth", International Journal of Control, vol. 33, edited by K. A. Gschneidner, Jr., J.-C. G. Bunzli, and V. Pecharsky, Elsevier Science, New York, 2003.

11] M. Pissas and G. Papavassiliou, "The phase diagram and magnetic properties of $\mathrm{La}_{1-x} \mathrm{Ca}_{x} \mathrm{MnO}_{3}$ compounds for $0 \leq x \leq$ 0.23", J. Phys.: Condens. Matter, vol. 16, pp. 6527-6540, 2004.

12] J. Hoffmann, P. Moschkau, S. Mildner, J. Norpoth, Ch. Jooss, L. $\mathrm{Wu}$, and Y. Zhu, "Effects of interaction and disorder on polarons in colossal resistance manganite $\operatorname{Pr}_{0.68} \mathrm{Ca}_{0.32} \mathrm{MnO}_{3}$ thin films", Mater. Res. Express, vol. 1, pp. 046403, 2014.

[13] J. Przybytek, J. Fink-Finowicki, R. Puzniak, A. Shames, V. Markovich, D. Mogilyansky, and G. Jung, "Robust Random Telegraph Conductivity Noise in Single Crystals of Ferromagnetic Insulating Manganite $\mathrm{La}_{0.86} \mathrm{Ca}_{0.14} \mathrm{MnO}_{3}$, Phys. Rev. B, vol. 95, pp. 125101, 2017.

14] P. G. Radaelli, G. Iannone, M. Marezio, H. Y. Hwang, S-W. Cheong, J. D. Jorgensen and D. N. Argyriou, "Structural effects on the magnetic and transport properties of perovskite $\mathrm{A}_{1-x} \mathrm{~A}_{x}^{\prime} \mathrm{MnO}_{3}(x=0.25,0.30) "$, Phys. Rev. B, vol. 56, pp. 8265 $-8276,1997$.

[15] D. Emin, "Current-driven threshold switching of a small polaron semiconductor to a metastable conductor", Phys. Rev. B, vol. 74, pp. 035206, 2008.

[16] D. Emin, "Theory of MeyerNeldel compensation for adiabatic charge transfer", Monatsh Chem., vol. 144, pp. 3-10, 2013.

Received 19 March 2019 